\title{
$\mathrm{Cr}-$ 및 $\mathrm{Ni}-$ 소스/드레인 쇼트키 박막 트랜지스터의 장벽 특성에 대한 실험 및 모델링 연구
}

\author{
정지철 ${ }^{1}$, 문경숙 ${ }^{2, a}$, 구상모 $^{1}$ \\ 1 광운대학교 전자재료공학과 \\ 2 경원대학교 수학정보학과
}

\section{Experimental and Simulation Study of Barrier Properties in Schottky Barrier Thin-Film Transistors with $\mathrm{Cr}^{-}$and $\mathrm{Ni}^{-}$- Source/Drain Contacts}

\author{
Ji-Chul Jung ${ }^{1}$, Kyoung-Sook Moon ${ }^{2, a}$, and Sang-Mo Koo ${ }^{1}$ \\ ${ }^{1}$ Department of Electronic Materials Engineering, Kwangwoon University, Seoul 139-701, Korea \\ ${ }^{2}$ Department of Mathematics and Information, Kyungwon University, Seongnam 461-701, Korea
}

(Received September 14, 2010; Accepted September 19, 2010)

\begin{abstract}
By improving the conducting process of metal source/drain (S/D) in direct contact with the channel, schottky barrier metal-oxide-semiconductor field effect transistors (SB MOSFETs) reveal low extrinsic parasitic resistances, offer easy processing and allow for well-defined device geometries down to the smallest dimensions. In this work, we investigated the arrhenius plots of the SB MOSFETs with different S/D schottky barrier (SB) heights between simulated and experimental current-voltage characteristics. We fabricated SB MOSFETs using difference S/D metals such as $\mathrm{Cr}\left(\Phi_{\mathrm{Cr}} \tilde{} 4.5 \mathrm{eV}\right)$ and $\mathrm{Ni}$ $\left(\Phi_{\mathrm{Ni}} \sim 2.2 \mathrm{eV}\right)$, respectively. Schottky barrier height $\left(\Phi_{B}\right)$ of the fabricated devices were measured to be $0.25^{\sim} 0.31 \mathrm{eV}(\mathrm{Cr}-\mathrm{S} / \mathrm{D}$ device$)$ and $0.16^{\sim} 0.18 \mathrm{eV}(\mathrm{Ni}-\mathrm{S} / \mathrm{D}$ device$)$, respectively in the temperature range of $300 \mathrm{~K}$ and $475 \mathrm{~K}$. The experimental results have been compared with 2-dimensional simulations, which allowed bandgap diagram analysis.
\end{abstract}

Keywords: Schottky barrier height, Temperature, Ni-S/D device, Cr-S/D device

\section{1. 서 론}

반도체 소자의 크기가 수십 나노미터 영역에 접어 들면서 기존의 실리콘 트랜지스터 소자의 동작 특성 을 제한하는 물리적 한계를 극복하기 위하여 다양한 기술들이 연구되고 있다. 그 중 쇼트키 장벽을 이용 한 접합 방법은 단채널 효과를 억제할 수 있고, 채널 실리콘 층에 낮은 도핑이 가능하여 on/off 동작의 향 상을 기대하며, 실리사이드의 얕은 접합 에 의한 기 생 저항 및 축전 용량을 감소시킬 수 있다 $[1,2]$. 또

a. Corresponding author; ksmoon@kyungwon.ac.kr
한, 터널링 효과에 의한 소자의 동작속도 향상과 저 온 공정의 금속 게이트 및 고 유전율 절연막을 이용 하므로 기존의 실리콘 기반 MOSFET (metal-oxidesemiconductor field effect transistors)에 비해서는 우 수한 동작 성능을 구현할 수 있는 장점이 있다 [3]. $\mathrm{SB}$ (schottky barrier) MOSFETs은 실리사이드의 면 저항은 불순물을 도핑한 실리콘보다 훨씬 작은 값을 가지므로 얕은 접합으로 인한 기생저항을 크게 줄이 는 특징을 나타내고 있다.

본 논문에서는 금속 물질인 $\mathrm{Cr}\left(\Phi_{\mathrm{Cr}} \tilde{} 4.5 \mathrm{eV}\right), \mathrm{Ni}$ $\left(\Phi_{\mathrm{Ni}} \sim 5.2 \mathrm{eV}\right)$ 을 각각 다른 $\mathrm{SOI}$ 기판에 증착하여 제작 


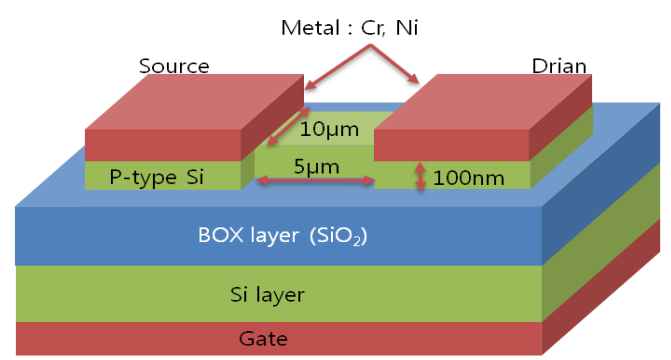

$\uparrow \uparrow \uparrow \uparrow \uparrow \uparrow \underset{\text { Temperature }}{\uparrow \uparrow \uparrow \uparrow \uparrow \uparrow}$

Fig. 1. Using SB MOSFET in experimental and simulation.

한 SB MOSFETs (그림 1)을 이용하였고, 온도에 따 른 전기적인 특성을 비교분석 하였다. $300 \mathrm{~K} \sim 475 \mathrm{~K}$ 온도에서 측정된 실험적인 결과와 시뮬레이션 결과를 arrhenius plot과 밴드갭 다이어그램 (band-gap diagram)로 분석하였고, 열전자 방출 (thermionic emission model)과 전기장 방출 (field emission model)로 설명하였다. 온도가 증가하면 $\mathrm{Cr}-\mathrm{S} / \mathrm{D}$ device는 온도가 증가할수록 유효전위장벽이 감소되 고 $(0.25 \sim 0.31 \mathrm{eV}), \mathrm{Ni}-\mathrm{S} / \mathrm{D}$ device는 온도가 증가할 수록 유효전위장벽이 증가한다 $(0.13 \sim 0.18 \mathrm{eV})$.

\section{2. 실험 방법}

$\mathrm{SB}$ MOSFET의 제작은 SOI (silicon on insulator) 웨이퍼를 기초로 하여, top-down approach 방식으로 제작하였다. 실리콘 $(\mathrm{Si})$ 은 $\mathrm{P}-\mathrm{type}$ 이고 약 $1017 \mathrm{~cm}^{-3}$ 정 도 도핑되었다. Si etchant를 사용하여 활성화 영역을 제작하였고, 채널의 폭은 $10 \mu \mathrm{m}$, 길이는 $5 \mu \mathrm{m}$ 이고 높 이는 $100 \mathrm{~nm}$ 이다. E-beam evaporator로 각각의 웨이 퍼에 금속 물질인 $\mathrm{Cr}$ 과 $\mathrm{Ni}$ 을 $100 \mathrm{~nm}$ 씩 source와 drain 영역에 증착하였고, lift-off 공정을 거쳐 $\mathrm{Cr}-\mathrm{S} / \mathrm{D}$ device와 $\mathrm{Ni}-\mathrm{S} / \mathrm{D}$ device를 제작하였다 (그림 1).

열 특성을 측정하기 위하여 $300 \mathrm{~K} \sim 475 \mathrm{~K}$ 온도를 $25 \mathrm{~K}$ 씩 증가시키며 소자에 가해주었고, $0 \sim 5 \mathrm{~V}$ 의 drain 전압과 $0 \mathrm{~V}$ 의 gate 전압을 인가하여 전류-전압 특성을 측정하였다.

측정한 자료는 식 (1)에 나타난 열전자 방출 이론 (thermionic emission)으로 유효전위장벽의 변화를 분 석하였다 [4].

$$
I=A_{d} A^{*} T^{2} \exp \left(\frac{-q \Phi_{B}}{k T}\right) \Rightarrow \Phi_{B}=-\frac{k T}{q} \ln \left(\frac{I}{A_{d} A^{*} T^{2}}\right)
$$
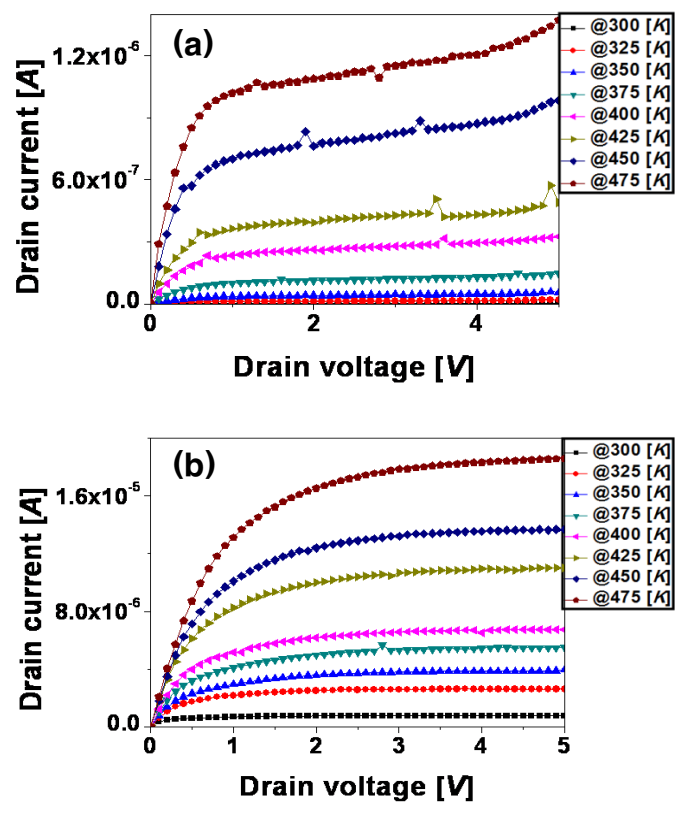

Fig. 2. Drain voltage-drain current curve in $300 \mathrm{~K}$ $475 \mathrm{~K}$ of temperature; (a) $\mathrm{Cr}-\mathrm{S} / \mathrm{D}$ device and (b) $\mathrm{Ni}-\mathrm{S} / \mathrm{D}$ device.

$\mathrm{A}$ 는 채널의 단면적 $\left(10^{-12} \mathrm{~m}^{2}\right), \mathrm{A}^{*}$ 는 Richardson 상수 $\left(\mathrm{Am}^{-2} \mathrm{~K}^{-2}\right), \quad \mathrm{T}$ 는 절대온도 $(\mathrm{K}), \mathrm{q}$ 는 전하량 $\left(1.6 \times 10^{-19} \mathrm{C}\right), \mathrm{k}$ 는 Boltzmann 상수 $\left(1.38 \times 10^{-23} \mathrm{JK}^{-1}\right)$, 그리고 $\Phi B$ 는 유효전위장벽이다.

시뮬레이션으로는 $0.1 \mathrm{~V}$ 의 drain 전압과 $0 \mathrm{~V}$ 의 gate 전압을 인가하여 gate의 영향을 받지 않는 조건 으로 수행하였다. 온도는 실제 측정내용과 같은 조건 으로 수행하였고, Fermi-Dirac 모델, concentration and temperature dependent 모델, Klaassen 모델, Lombardi 모델, 그리고 Shockley-Read-Hall 모델을 사용하여 시뮬레이션 하였다. 식 (1)에 의해 도출된 arrhenius plot을 시률레이션 결과인 밴드갭 다이어그 램 (band-gap diagram)과 함께 분석하였다.

\section{3. 결과 및 고찰}

온도가 증가할 경우, $\mathrm{SB}$ MOSFETs의 drain 전류 는 증가하였고, 전류가 포화 (saturation)되는 drain 전압위치는 양의 방향으로 이동하였다.

$\mathrm{Cr}-\mathrm{S} / \mathrm{D}$ device의 최대 drain 전류는 $3.74 \times 10^{-9} \mathrm{~A}$ 에 서 $1.37 \times 10^{-6} \mathrm{~A}$ 까지 비교적 비례적으로 증가하였고, $\mathrm{Ni}-\mathrm{S} / \mathrm{D}$ device의 최대 drain 전류는 $7.71 \times 10^{-7} \mathrm{~A}$ 에서 


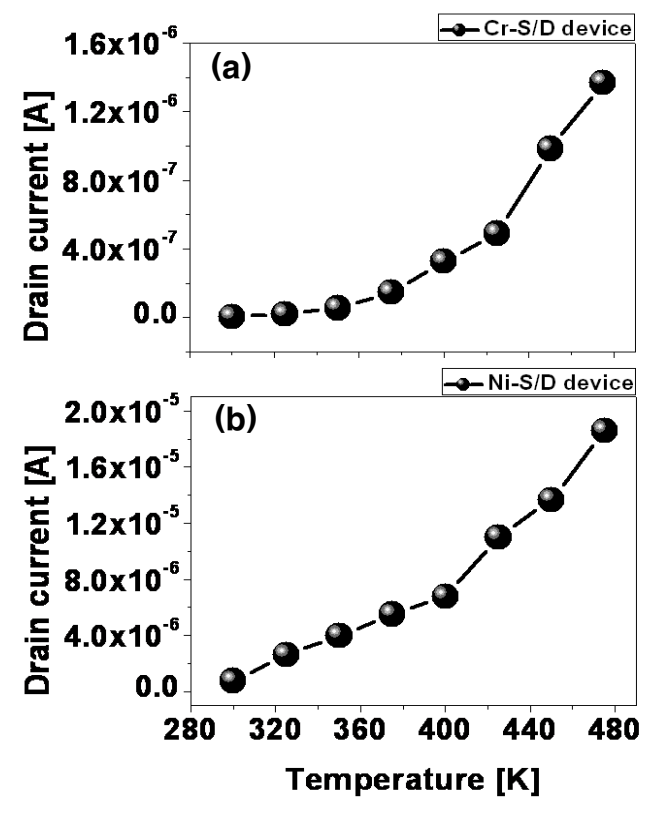

Fig. 3. Increased drain current by changing temperature from $300 \mathrm{~K}$ to $475 \mathrm{~K}$; (a) $\mathrm{Cr}-\mathrm{S} / \mathrm{D}$ device and (b) $\mathrm{Ni}-\mathrm{S} / \mathrm{D}$ device.

$1.85 \times 10^{-5} \mathrm{~A}$ 까지 선형적으로 증가하였다. 이는 그림 2 와 그림 3 에서 확인할 수 있다. (a)는 $\mathrm{Cr}-\mathrm{S} / \mathrm{D}$ device 의 전류-전압 특성을 나타내었고 (b)에서는 $\mathrm{Ni}-\mathrm{S} / \mathrm{D}$ device의 전류-전압 특성을 나타내었다. 식 (1)을 보 면 전류는 전위장벽보다 온도의 영향을 더 많이 받음 을 알 수 있다. 또한 측정된 전류-전압 특성과 열전 자 방출 (thermionic emission)이론으로 arrhenius plot을 도출할 수가 있다. 그림 4(a)는 $\mathrm{Cr}-\mathrm{S} / \mathrm{D}$ device 의 arrhenius plot을 나타내고 온도가 증가할수록 유 효전위장벽이 감소되는 것을 알 수 있다. Drain 전압 이 $0.5 \mathrm{~V}$ 인 경우, 상온 $(300 \mathrm{~K})$ 에서는 약 $0.31 \mathrm{eV}$ 이 고 $475 \mathrm{~K}$ 온도에서는 약 $0.26 \mathrm{eV}$ 로 $0.05 \mathrm{eV}$ 정도 감 소되었다. 그림 4(b)는 Ni-S/D device의 arrhenius plot을 나타낸 그래프이고 온도가 증가할수록 유효전 위장벽이 증가하는 것을 알 수 있다. Drain 전압이 $0.5 \mathrm{~V}$ 인 경우, 상온 $(300 \mathrm{~K})$ 에서는 약 $0.16 \mathrm{eV}$ 이고 $475 \mathrm{~K}$ 온도에서는 약 $0.18 \mathrm{eV}$ 로 약 $0.02 \mathrm{eV}$ 정도 증 가되었다. 두 소자의 공통된 특성은 drain 전압이 증 가하면 유효전위장벽이 감소하는 것이다. 이는 유효 전위장벽은 전계의 영향을 받았다는 것을 의미한다.

그림 5 의 측정된 결과와 그림 4 의 측정된 결과를 비교분석하면, $\mathrm{Cr}-\mathrm{S} / \mathrm{D}$ device 경우, 온도가 증가할수 록 유효전위자벽도 감소하고 $-\ln \left(\mathrm{I} / \mathrm{T}^{2}\right)$ 도 감소한다.
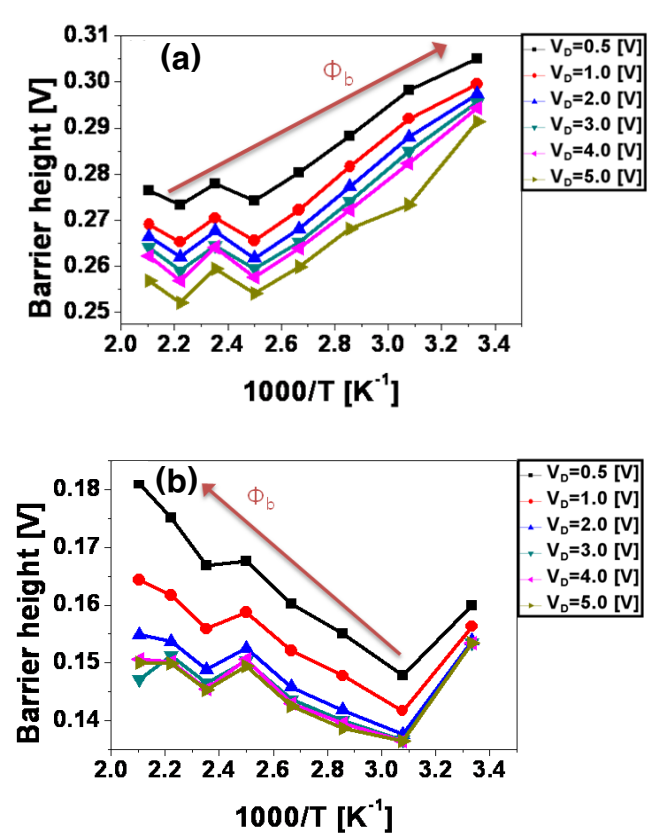

Fig. 4. Extracted schottky barrier height for different applied drain bias and zero bias gate; (a) $\mathrm{Cr}-\mathrm{S} / \mathrm{D}$ device and (b) $\mathrm{Ni}-\mathrm{S} / \mathrm{D}$ device.
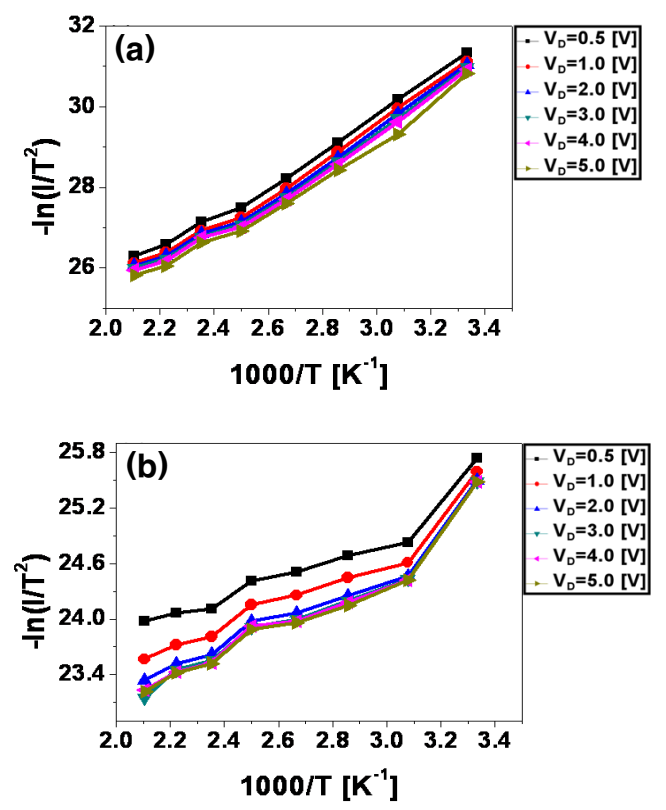

Fig. 5. Experimental and calculated arrhenius plot for different applied drain bias and zero bias gate; (a) $\mathrm{Cr}-\mathrm{S} / \mathrm{D}$ device and (b) Ni-S/D device.

이는 식 (1) 중 온도에 비례한 $\mathrm{kT} / \mathrm{q}$ 의 영향을 받지 않고 소자내부저항 (series resistance)에 영향을 받았 

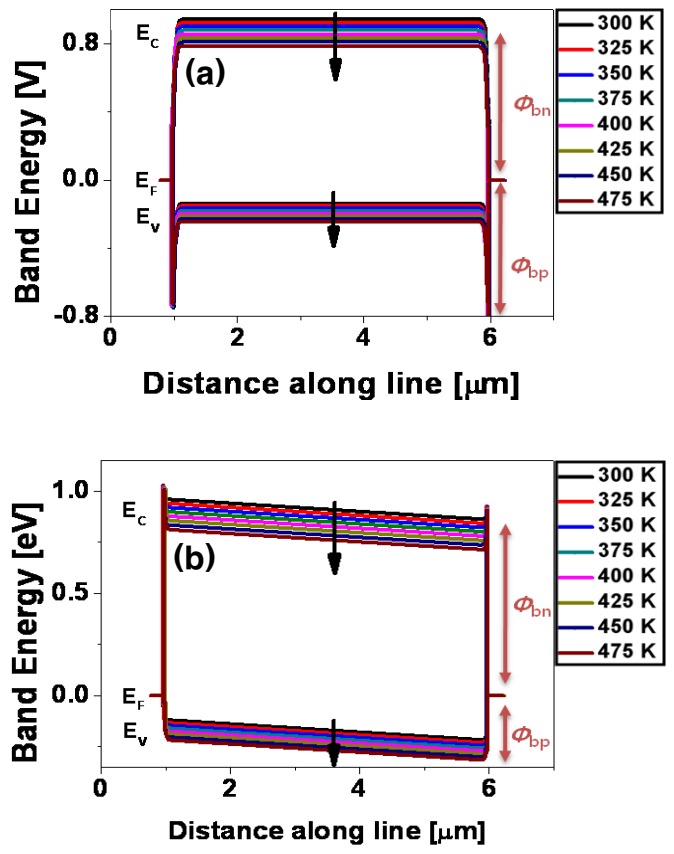

Fig. 6. Simulated band-gap diagram for different temperature; (a) $\mathrm{Cr}-\mathrm{S} / \mathrm{D}$ device and (b) Ni-S/D device.

음을 의미한다. 그에 비해 $\mathrm{Ni}-\mathrm{S} / \mathrm{D}$ device 경우, 온도 가 증가할수록 유효정위장벽은 증가하지만 $-\ln \left(\mathrm{I} / \mathrm{T}^{2}\right)$ 은 감소한다. 즉, 온도에 비례한 $\mathrm{kT} / \mathrm{q}$ 의 영향을 받았 음을 알 수 있다.

$\mathrm{Cr}^{-}$및 $\mathrm{Ni}-\mathrm{S} / \mathrm{D}$ device의 밴드갭 다이어그램 (bandgap diagram)은 시률레이션으로 분석하였고, 그 결과 를 그림 6에 나타내었다. 온도가 증가하면, 두 소자의 전도대 (conduction band)와 가전자대 (valance band) 는 낮은 밴드 에너지 (band energy)를 갖게 되고 전 도대 (conduction band)가 가전자대 (band energy)에 비해 더 많이 변화한다. 외인성 반도체 (extrinsic semiconductor)의 Fermi 에너지 준위는 온도가 증가 할수록 $\mathrm{E}_{\mathrm{g}} / 2$ 지점으로 다가가게 되고 에너지 갭 $\left(\mathrm{E}_{\mathrm{g}}\right)$ 은 미세하게 감소하게 된다. 온도에 영향을 받는 $\mathrm{Ni}-\mathrm{S} / \mathrm{D}$ device의 유효전위장벽은 그림 6 에서 $\Phi_{\mathrm{bp}}$ 인 것을 알 수 있고, $\mathrm{P}$-type $\mathrm{Si}$ 의 다수 캐리어인 정공 (hole)이 느끼는 전위장벽이 높아진다. $\mathrm{Cr}-\mathrm{S} / \mathrm{D}$ device 는 $300 \mathrm{~K} \sim 475 \mathrm{~K}$ 온도 내에서 소자내부저항 (series resistance)에 영향을 받는다.

\section{4. 결 론}

본 연구에서는 $\mathrm{SB}$ MOSFETs $(\mathrm{Cr}-$ 및 $\mathrm{Ni}-\mathrm{S} / \mathrm{D}$ device)의 유효전위장벽과 열-전기적 특성을 실험 결 과와 시뮬레이션 결과를 통해 확인하였다. $\mathrm{Cr}-\mathrm{S} / \mathrm{D}$ device는 온도가 증가할수록 유효전위장벽이 감소하 였고 이를 $300 \mathrm{~K} \sim 475 \mathrm{~K}$ 온도 내에서는 소자내부저 항 (series resistance)에 영향을 받았다고 해석하였다. 반면, Ni-S/D device는 $300 \mathrm{~K} \sim 475 \mathrm{~K}$ 온도 내에서 온도가 증가할수록 유효전위장벽이 증가하였고, 이를 열전자 방출 (thermionic emission)이론과 전기장 방 출 (field emission model)이론으로 해석하였다. 추가 적으로 시률레이션 결과인 밴드갭 다이어그램 (band-gap diagram)으로 해석하였고, $\mathrm{Ni}-\mathrm{S} / \mathrm{D}$ device 의 유효전위장벽은 정공 (hole)이 이동할 때 느끼는 전위장벽임을 확인하였다.

\section{감사의 글}

본 연구는 2008년 경원대학교 지원에 의한 결과이 며, 또한 2009년 정부 (교육과학기술부)의 재원으로 한국연구재단의 지원을 받아 수행된 연구입니다 (2009-0066544).

\section{REFERENCES}

[1] J. Knoch, M. Zhang, Q. Tzhao, S. Lenk, and S. Mantl, Appl. Phys. Lett. 87, 263505 (2005).

[2] L. E. Calvet, H. Luebben, M. A. Reed, C. Wang, J. P. Snyder, and J. R. Tucker, Appl. Phys. Lett. 91, 757 (2002).

[3] M. Jang, J. Oh, S. Maeng, W.-J. Cho, S. Lee, K. Kang, and K. Park, Appl. Phys. Lett. 83, 2611 (2003).

[4] E. Dubois and G. Larrieu, Appl. Phys. Lett. 96, 1 (2004). 